\title{
Erratum to: The phenotypic spectrum of organic acidurias and urea cycle disorders. Part 1: the initial presentation
}

Stefan Kölker ${ }^{1}$ - Angeles Garcia Cazorla ${ }^{2}$ - Vassili Valayannopoulos ${ }^{3} \cdot$ Allan M. Lund $^{4}$. Alberto B. Burlina ${ }^{5}$. Jolanta Sykut-Cegielska ${ }^{6}$. Frits A. Wijburg ${ }^{7}$ Elisa Leão Teles ${ }^{8}$. Jiri Zeman ${ }^{9}$ - Carlo Dionisi-Vici ${ }^{10}$ - Ivo Barić $^{11}$ • Daniela Karall ${ }^{12}$. Persephone Augoustides-Savvopoulou ${ }^{13}$ • Lise Aksglaede ${ }^{4}$. Jean-Baptiste Arnoux ${ }^{3}$. Paula Avram $^{14}$ • Matthias R. Baumgartner ${ }^{15}$. Javier Blasco-Alonso ${ }^{16}$ - Brigitte Chabrol ${ }^{17}$. Anupam Chakrapani ${ }^{18}$ - Kimberly Chapman ${ }^{19}$ - Elisenda Cortès i Saladelafont ${ }^{2} \cdot$ Maria L. Couce $^{20}$. $^{2}$ Linda de Meirleir ${ }^{21}$ - Dries Dobbelaere ${ }^{22}$ - Veronika Dvorakova ${ }^{9}$ - Francesca Furlan ${ }^{5}$. Florian Gleich $^{1}$ - Wanda Gradowska ${ }^{23}$ • Stephanie Grünewald ${ }^{24}$ • Anil Jalan ${ }^{25}$ • Johannes Häberle ${ }^{15}$. Gisela Haege $^{1}$ - Robin Lachmann ${ }^{26}$ • Alexander Laemmle ${ }^{15}$ - Eveline Langereis ${ }^{7}$.

Pascale de Lonlay $^{3}$ • Diego Martinelli ${ }^{10}$ - Shirou Matsumoto ${ }^{27}$. Chris Mühlhausen ${ }^{28}$. Hélène Ogier de Baulny ${ }^{29}$. Carlos Ortez ${ }^{2}$ Luis Peña-Quintana ${ }^{31} \cdot$ Danijela Petković Ramadža $^{30}$. Esmeralda Rodrigues $^{8} \cdot$ Sabine Scholl-Bürgi $^{12}$ • Etienne Sokal ${ }^{32}$ - Christian Staufner ${ }^{1}$. Marshall L. Summar ${ }^{19}$ • Nicholas Thompson ${ }^{24}$. Roshni Vara ${ }^{33}$ • Inmaculada Vives Pinera ${ }^{34}$. John H. Walter ${ }^{35}$ - Monique Williams ${ }^{36}$ - Peter Burgard ${ }^{1}$

Published online: 16 June 2015

(C) SSIEM 2015

Erratum to: J Inherit Metab Dis DOI 10.1007/s10545-015-9839-3

The name of Angeles Garcia Cazorla is not correctly stated. It must read Angeles Garcia-Cazorla and A. Garcia-Cazorla

The online version of the original article can be found at http://dx.doi.org/ 10.1007/s10545-015-9839-3.

Stefan Kölker

Stefan.Koelker@med.uni-heidelberg.de

1 Department of General Pediatrics, Division of Inherited Metabolic Diseases, University Children's Hospital Heidelberg, Im Neuenheimer Feld 430, D-69120 Heidelberg, Germany

2 Servicio de Neurologia and CIBERER, ISCIII, Hospital San Joan de Deu, Barcelona, Spain 
3 Hôpital Necker-Enfants Malades, Assistance Publique-Hôpitaux de Paris, Reference Center for Inherited Metabolic Disease, Necker-Enfants Malades University Hospital and IMAGINE Institute, Paris, France

4 Centre for Inherited Metabolic Diseases, Department of Clinical Genetics, Copenhagen University Hospital, Rigshospitalet, Copenhagen, Denmark

5 U.O.C. Malattie Metaboliche Ereditarie, Azienda Ospedaliera di Padova, Padova, Italy

6 Screening Department, Institute of Mother and Child, Warsaw, Poland

7 Department of Pediatrics, Academisch Medisch Centrum, Amsterdam, Netherlands

8 Unidade de Doenças Metabólicas, Serviço de Pediatria, Hospital de S. João, EPE, Porto, Portugal

9 First Faculty of Medicine, Charles University and General University of Prague, Prague, Czech Republic

10 U.O.C. Patologia Metabolica, Ospedale Pediatrico Bambino Gésu, Rome, Italy

11 School of Medicine, University Hospital Center Zagreb and University of Zagreb, Zagreb, Croatia

12 Clinic for Pediatrics I, Inherited Metabolic Disorders, Medical University of Innsbruck, Innsbruck, Austria

13 1st Pediatric Department, Metabolic Laboratory, General Hospital of Thessaloniki 'Hippocration', Thessaloniki, Greece

14 Institute of Mother and Child Care "Alfred Rusescu", Bucharest, Romania

15 Division of Metabolism and Children's Research Centre, University Children's Hospital Zurich, Steinwiesstraße 75, CH-8032 Zurich, Switzerland

16 Hospital Materno-Infantil (HRU Carlos Haya), Málaga, Spain

17 Centre de Référence des Maladies Héréditaires du Métabolisme, Service de Neurologie, Hôpital d'Enfants, CHU Timone, Marseilles, France

18 Birmingham Children's Hospital NHS Foundation Trust, Steelhouse Lane, Birmingham B4 6NH, UK
19 Children's National Medical Center, 111 Michigan Avenue, N.W., Washington, DC 20010, USA

20 Metabolic Unit, Department of Pediatrics, Hospital Clinico Universitario de Santiago de Compostela, Santiago de Compostela, Spain

21 University Hospital Vrije Universiteit Brussel, Bruxelles, Belgium

22 Centre de Référence des Maladies Héréditaires du Métabolisme de l'Enfant et de l'Adulte, Hôpital Jeanne de Flandre, Lille, France

23 Department of Laboratory Diagnostics, The Children's Memorial Health Institute, Warsaw, Poland

24 Metabolic Unit Great Ormond Street Hospital and Institute for Child Health, University College London, London, UK

25 N.I.R.M.A.N., Om Rachna Society, Vashi, Navi Mumbai, Mumbai, India

26 Charles Dent Metabolic Unit, National Hospital for Neurology and Neurosurgery, London, UK

27 Department of Pediatrics, Kumamoto University Hospital, Kumamoto City, Japan

28 Klinik für Kinder- und Jugendmedizin, Universitätsklinikum Hamburg-Eppendorf, Hamburg, Germany

29 Hôpital Robert Debré, Université de Paris, Paris, France

30 University Hospital Center Zagreb, Zagreb, Croatia

31 Hospital Universitario Materno-Infantil de Canarias, Unit of Pediatric Gastroenterology, Hepatology and Nutrition, University of Las Palmas de Gran Canaria, Las Palmas de Gran Canaria, Spain

32 Service Gastroentérologie and Hépatologie Pédiatrique, Cliniques Universitaires St Luc, Université Catholique de Louvain, Bruxelles, Belgium

33 Evelina Children's Hospital, St Thomas' Hospital, London, UK

34 Inborn Metabolic Disease Unit, Hospital Virgen de la Arrixaca de Murcia, El Palmar, Spain

35 Manchester Academic Health Science Centre, Willink Biochemical Genetics Unit, Genetic Medicine, University of Manchester, Manchester, UK

36 Erasmus MC-Sophia Kinderziekenhuis, Erasmus Universiteit Rotterdam, Rotterdam, Netherlands 